\title{
Feasibility study of imaging spectroscopy to monitor the quality of online welding
}

\author{
Jesús Mirapeix, ${ }^{*}$ P. Beatriz García-Allende, Adolfo Cobo, \\ Olga M. Conde, and José M. López-Higuera \\ Laboratorios I\&D Telecomunicaciones, Department of Electronics Technology, Systems and \\ Automation Engineering (TEISA), Grupo de Ingeniería Fotónica-Universidad de Cantabria, \\ Avda. Los Castros s/n, 39005 Santander, Spain \\ ${ }^{*}$ Corresponding author: jesus.mirapeix @ unican.es
}

Received 26 September 2008; revised 17 July 2009; accepted 29 July 2009; posted 3 August 2009 (Doc. ID 102114); published 13 August 2009

\begin{abstract}
An online welding quality system based on the use of imaging spectroscopy is proposed and discussed. Plasma optical spectroscopy has already been successfully applied in this context by establishing a direct correlation between some spectroscopic parameters, e.g., the plasma electronic temperature and the resulting seam quality. Given that the use of the so-called hyperspectral devices provides both spatial and spectral information, we propose their use for the particular case of arc welding quality monitoring in an attempt to determine whether this technique would be suitable for this industrial situation. Experimental welding tests are presented, and the ability of the proposed solution to identify simulated defects is proved. Detailed spatial analyses suggest that this additional dimension can be used to improve the performance of the entire system. (C) 2009 Optical Society of America

OCIS codes: $\quad 060.2370,300.2140,120.4630,350.3850$.
\end{abstract}

\section{Introduction}

By acquiring the information that comes from the interaction of light and materials, distinct spectroscopic techniques have been proposed to solve a wide range of problems in a variety of situations from biomedical research [1] to environmental monitoring [2], semiconductor processing [3], or the analysis of art or archeological findings [4]. An area in which this kind of noncontact solution has found great acceptance is the online quality monitoring of industrial processes, for example, in the food and fruit industry $[5,6]$. Recently, imaging spectroscopy has attracted great attention in this field, although it has been typically employed for remote sensing [7]. The ability of the so-called multispectral or hyperspectral devices to provide a two-dimensional data set with both spectral and spatial information can be a relevant asset within this framework, especially if the

0003-6935/09/244735-08\$15.00/0

(C) 2009 Optical Society of America material under analysis is transported by means of a conveyor belt. Some research has already dealt with quality monitoring in the food [8] and tobacco [9] industry by use of this approach with promising results.

Another good example of the necessity to use online quality monitoring systems in industrial environments can be found in both arc and laser welding. These processes are extensively used in the manufacture of airplanes, automobiles, and heavy components for nuclear power stations, to mention just a few examples for which quality requirements are in demand. Different approaches have been considered to solve the problem of detecting weld defects online, such as the analysis of acoustic emission produced during the process [10] or the use of machine vision [11] or thermographic [12] systems. However, nowadays it is difficult to find this kind of solution implemented in production stages, and typically weld coupons and nondestructive testing (NDT) techniques are chosen to ensure that quality standards are fulfilled. It is worth mentioning that an effective 
online monitoring system would benefit not only the identification of the appearance of defects but also possible implementation of real-time control of the welding system to try to prevent the defects.

Plasma optical spectroscopy has been thoroughly investigated within this framework, and several reports have shown that an online analysis is feasible, typically by considering simplifications to estimate the plasma electronic temperature $T_{e}[13,14]$. This parameter is known to exhibit a direct correlation to the quality of the associated seam and, therefore, the rapid variations of its profile are associated with the appearance of defects. However, there is still an uncertainty in terms of the plasma lines involved in the analysis, whose intensity is averaged through the line of observation determined by the system input optics. This makes it impossible to carry out a spatial analysis of the plasma column, which could be useful to select the optimum spectral bands in terms of defect discrimination.

We propose the use of imaging spectroscopy, not only to be employed as an online arc-welding quality monitoring system, but also to perform a spectral band and spatial selection within the plasma column that could also be used with the traditional spectroscopic approach. Experimental tests with simulated defects will show the feasibility of the proposed solution, and detailed spatial analysis of the plasma column will also be presented and discussed.

\section{Plasma Spectroscopy}

Plasma diagnostics applied to monitoring the quality of online welding typically relies on two main assumptions: the plasma is supposed to be optically thin and in local thermodynamic equilibrium (LTE) [15]. The former needs to be experimentally verified by checking that the selected emission lines are free from self-absorption [16]; LTE is typically assumed to be valid for arc-welding plasmas and most particularly for tungsten inert gas (TIG), also known as gas tungsten arc welding (GTAW) processes. This hypothesis allows characterization of the plasma temperature by means of the plasma electronic temperature $T_{e}$, and it implies that the collisional process is the dominant mechanism within the plasma. In addition, LTE also means that the gradients for both temperature and concentrations will be small, which is not valid in the vicinity of the cathode and anode [17]. Several authors have discussed the validity of assuming LTE for arc welding, establishing, for example, a lower threshold for the welding current of $35 \mathrm{~A}$ [18]. An accepted criterion to determine whether LTE can be accepted for a given plas$\mathrm{ma}$ is

$$
N_{e} \geq 1.6 \times 10^{12} T_{e}^{1 / 2}(\Delta E)^{3},
$$

where $N_{e}$ is the plasma electronic density and $\Delta E$ is the largest energy gap in the atomic energy level system.
If both conditions were fullfilled, $T_{e}$ could be obtained by a variety of spectroscopic techniques, with Eq. (2) being the most common approximation to be found in the literature applied to online arc-welding quality monitoring [19]:

$$
T_{e}=\frac{E_{m}(2)-E_{m}(1)}{k \times \ln \left[\frac{E_{m}(1) I(1) A(2) g_{m}(2) \lambda(1)}{E_{m}(2) I(2) A(1) g_{m}(1) \lambda(2)}\right]},
$$

where $E_{m}$ is the upper level energy of the emission line with relative intensity $I, A$ is the transition probability, $g_{m}$ is the statistical weight, and $\lambda$ is the associated wavelength. This approximation gives rise to temperature values with a significant uncertainty, even if there is adequate difference between the upper level energies of the chosen emission lines. In this regard, a more precise estimation can be obtained by the Boltzmann-plot method:

$$
\ln \left(\frac{I_{\mathrm{mn}} \lambda_{\mathrm{mn}}}{A_{\mathrm{mn}} g_{m}}\right)=\ln \left(\frac{h c N}{Z}\right)-\frac{E_{m}}{k T_{e}} .
$$

However, in this case several emission lines must be included in the calculations, which makes this solution less efficient in terms of the computational performance of the required online analysis system. The known correlation between the $T_{e}$ profiles and the associated seam quality $[13,14,20]$ has been recently analyzed for the line-to-continuum method [21], but in this preliminary study not all the profiles exhibit the same defect detection ability, depending on the chosen spectral band.

In all these methods a single spatially averaged $T_{e}$ value is typically determined for the entire plasma column, since the plasma radiation is usually collected by means of a collimator [20] or an optical fiber [22] arranged at a given distance from the plasma axis. In contrast, spatially resolved emission spectroscopy techniques allow one to obtain a detailed map of the temperature distributions within the plasma, which requires a deconvolution process to achieve the desired spatial resolution, e.g., the Abel inversion [23]. This method is obviously more sophisticated, but it cannot be used for real-time analysis given its processing requirements.

Although the spectroscopic approach has proved to be an effective solution within this framework, some problems can still appear during practical implementations. For example, if a complex system with several weld heads needs to be monitored, the costs associated with the spectrometer should be reduced by selecting low-cost devices. However, a lower spectral resolution will provoke a higher uncertainty in terms of emission line identification, i.e., the association of a given emission line with its chemical species. In addition, it could be highly interesting to determine which spectral bands are more sensitive to the identification of different weld defects. The use of imaging spectroscopy and its ability to acquire 
not only the plasma spectral information but also its spatial information will prove to have great potential in this regard.

\section{Hyperspectral Emission Imaging of Welding Plasma}

The acquisition of hyperspectral images of the plasma formed in welding processes, with a plasma spectrum measured for each spatial element in the plasma column, is illustrated in Fig. 1. The captured data represent a three-dimensional data set, the so-called image cube, defined by spatial $x$ and $y$ and wavelength $\lambda$ coordinates. Since standard twodimensional detectors can register only the position and intensity of radiation at the same time, the spatial information across a given line ( $x$ axis with a specified length to cover the plasma column and a small but finite width) and the spectral information (wavelength and intensity) are measured simultaneously. One dimension of the detector constitutes a spatial line image and the other dimension measures the spectrum for each line pixel. Scanning the third dimension of the image cube, the second spatial dimension $y$ is accomplished by movement of the object, i.e., the displacement of the torch and, consequently, the plasma column along the seam.

A photograph of the measurement setup used in our laboratory is shown in Fig. 2. The hyperspectral imaging system can be observed on the left-hand side. It is divided into two modules: sensor and optics. The latter includes the equipment known as ImSpector, a commercial product of Spectral Imaging, Ltd. (Oulu, Finland), which has been used as an imaging spectrograph and a C-mount lens. The dispersing element of the spectrograph is a volume type holographic transmission grating, which is used in a prism-grating-prism (PGP) structure and provides high diffraction efficiency and good spectral linearity [24]. Since measurements were intended to be performed in the visible-NIR range, the V10E version of ImSpector, whose spectral range is from 400 to $1000 \mathrm{~nm}$, was chosen. Navitar Zoom 7000 (Navitar, Rochester, New York) is the selected objective lens, whose focusing range has been manually controlled to cover the entire width of the plasma column. The sensor module includes the high-performance monochrome digital camera Pixelink PL-A741 MV (Aegis Electronic Group, Gilbert, Arizona), whose spectral range varies from 350 to $1000 \mathrm{~nm}$ with a resolution of $640 \times 480$ pixels (spatial and spectral dimensions, respectively). The communication interface between camera and control and acquisition computer is IEEE1394.

The wavelength calibration process, which defines the spectral axis, is necessary once the system setup has been arranged, before beginning the acquisition of spectral images.The calibration process is

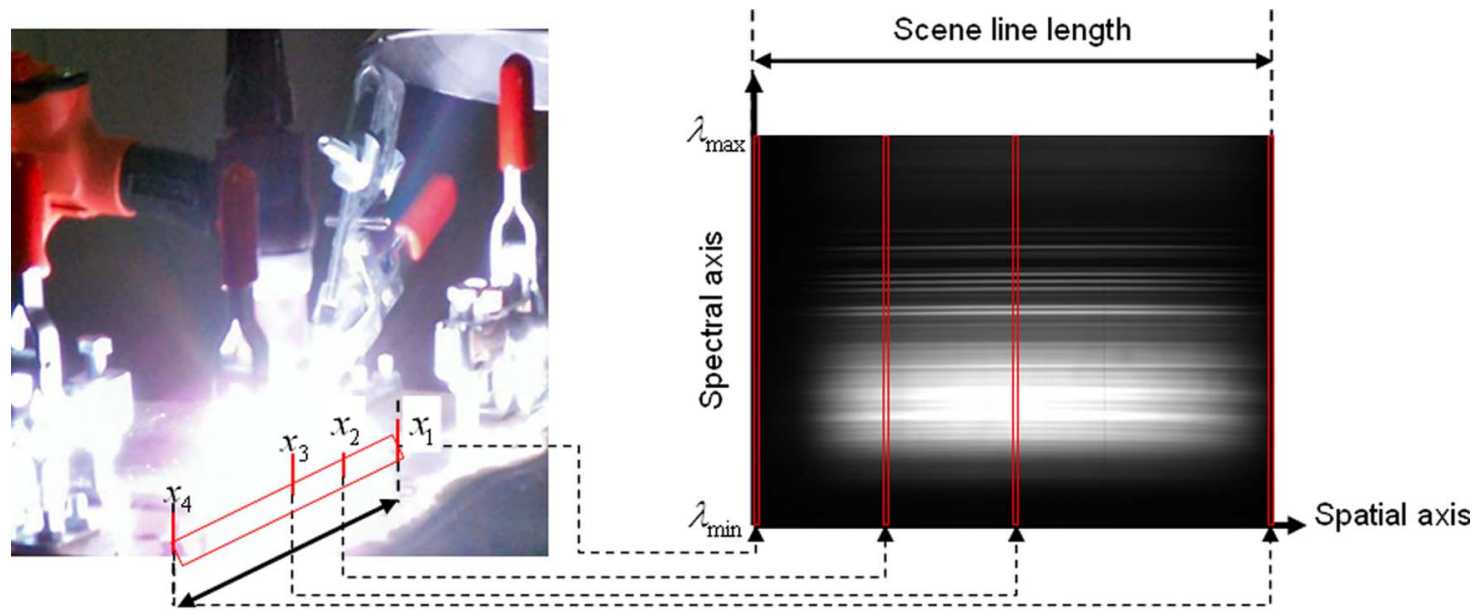

Scene line length

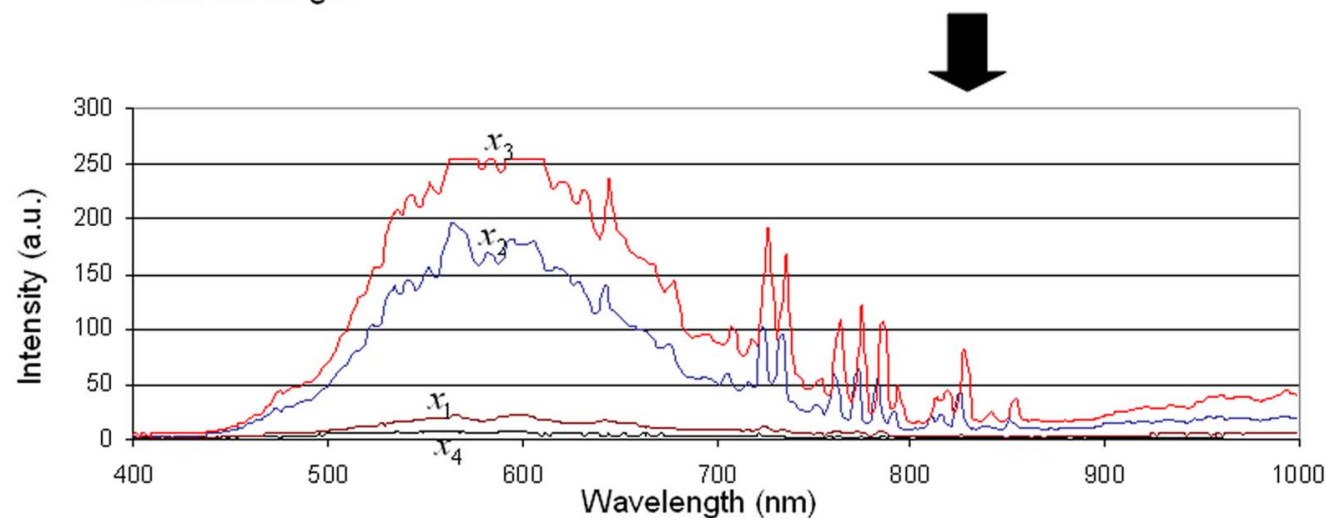

Fig. 1. (Color online) Schematic representation of the acquisition of hyperspectral images of the welding plasma column. 


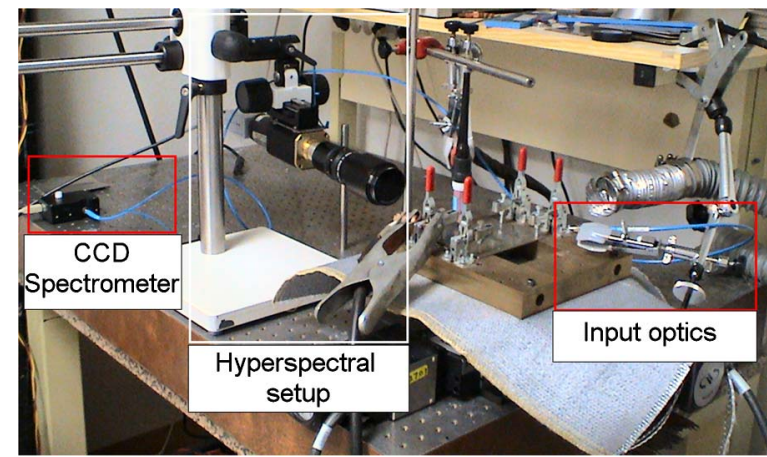

Fig. 2. (Color online) Experimental setup with the imaging (center) and traditional spectroscopic (left and right) systems.

performed using two different light sources whose emission wavelengths are previously known, e.g., a $670 \mathrm{~nm}$ laser and a $\mathrm{Hg}-\mathrm{Ar}$ lamp with multiple emission lines.

The right-hand side of Fig. $\underline{2}$ depicts the setup that is used to perform the experimental welding tests. The tests were carried out with an arc-welding [tungsten inert gas (TIG)] system that includes a Kemppi Mastertig 2200 (General Welding Supplies, Cheshire, UK) power source, a welding torch (Kemppi TTC 220), and a positioning system (Newport MM4005 controller and two MTM100PP1 stages; Newport Corporation, Irvine, California). Since hyperspectral images must be captured without variations in conditions such as the distance between target and objective lens or the lens focal length, the welding torch and, therefore, the plasma column must remain fixed. The AISI-304 stainless steel plates are fastened to the positioning system and controlled by a PC; Table 1 shows the chemical composition of this material. In addition, argon was used as the shielding gas with a constant flow rate of $12 \mathrm{l} / \mathrm{min}$ in standard operating conditions.

An additional acquisition system was also used during the welding tests to facilitate a direct correlation between imaging and conventional spectroscopic techniques. In the latter the plasma radiation is spatially integrated by means of input optics and a CCD spectrometer. The system consists of an optical fiber (P50-2-UVVIS, $2 \mathrm{~m}$ long and $50 \mu \mathrm{m}$ core diameter; Ocean Optics, Dunedin, Florida) attached to a CCD spectrometer (Ocean Optics USB2000). The other

Table 1. Chemical Composition of AISI-304

\begin{tabular}{cc}
\hline \multicolumn{3}{c}{ AISI-304 } \\
\hline Element & \% Participation \\
\hline $\mathrm{Fe}$ & - \\
$\mathrm{Cr}$ & $18-20$ \\
$\mathrm{Ni}$ & $8-10.5$ \\
$\mathrm{Mn}$ & $2(\max )$ \\
$\mathrm{Si}$ & $1(\max )$ \\
$\mathrm{C}$ & $0.08(\max )$ \\
$\mathrm{P}$ & $0.04(\max )$ \\
$\mathrm{S}$ & $0.03(\max )$ \\
\hline
\end{tabular}

end of the fiber was focused on the plasma axis (between the electrode tip and the plate). The distance between the fiber end and the plasma axis was approximately $14 \mathrm{~cm}$. An example of a plasma spectrum captured by this additional system is presented in Fig. 3. In this example some of the emission lines have been identified to illustrate the chemical composition of the plasma under analysis.

\section{Experimental Issues}

An initial set of experiments was conducted to acquire the spectra with the systems mentioned above during standard welding operation conditions. Two examples of seams performed with constant welding currents of 26 and $40 \mathrm{~A}$ are presented in Fig. 4. Figures 4(a) and 4(c) show the images obtained with the imaging spectroscopy setup; Figs. $4(\mathrm{~b})$ and $4(\mathrm{~d})$ show the associated spectra captured by means of the conventional approach. It is worth mentioning that the spectral range is different in both situations: from 400 to approximately $1000 \mathrm{~nm}$ in the hyperspectral images (vertical axis), and from 195 to $535 \mathrm{~nm}$ in Figs. 4(b) and 4(d). As expected, the plasma radiation exhibits increased intensity with higher welding currents, which can be clearly appreciated in this example. A detail of the plasma column can be seen in Fig. 4(e), where several plasma emission lines can be found.

Although different approaches could be considered, it does not seem neccesary with this new solution to perform a plasma emission line identification stage, i.e., the association of each emission line with its corresponding chemical species, in terms of the ability of the system to detect weld defects. On this point it should be noted that, initially, the main purpose of this study was to determine whether this kind of solution could be used in that regard. Given the kind of images acquired by the system, it seems that a simple image processing approach would be more efficient; apart from the lack of optical resolution (of the order of $2.8 \mathrm{~nm}$ ), which would make it difficult to perform a reliable line identification [20].

A good example to illustrate the performance of the proposed approach is exhibited in Fig. 5, where a seam was performed with a constant welding current

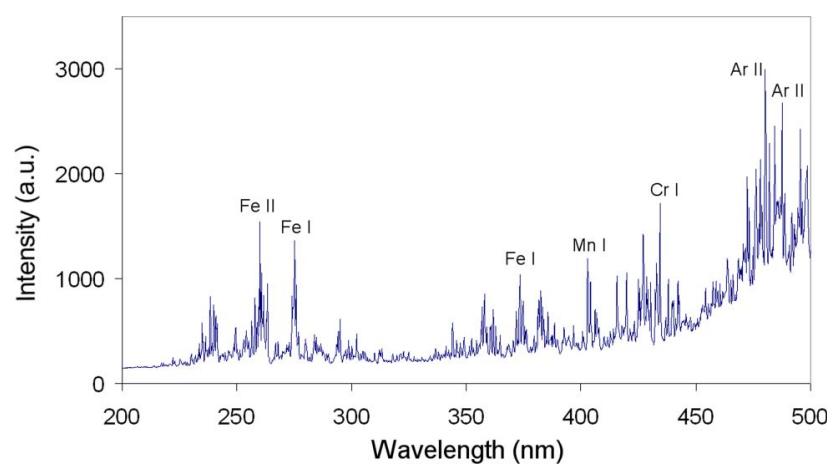

Fig. 3. (Color online) Example of plasma spectrum captured by the CCD spectrometer with some emission lines associated with their corresponding species. 


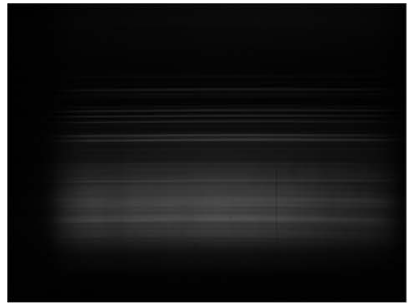

(a)

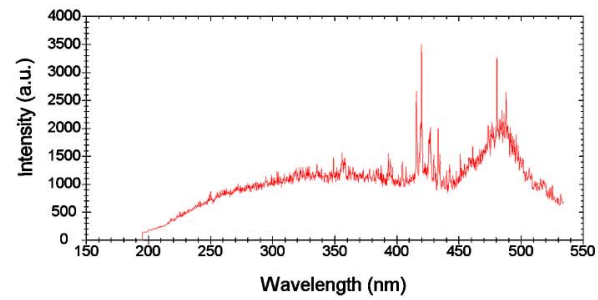

(d)

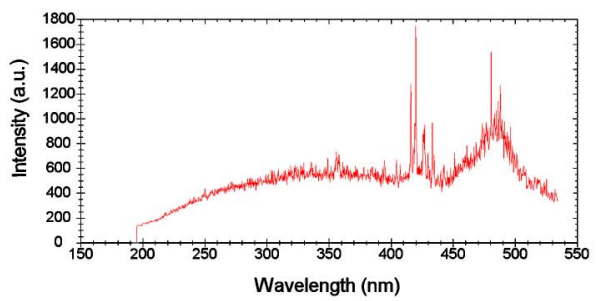

(b)

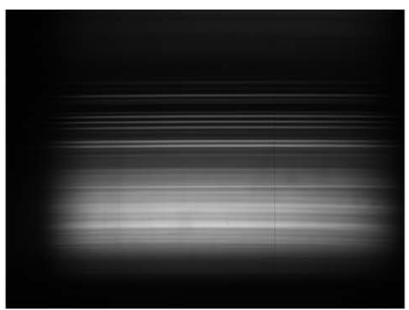

(c)

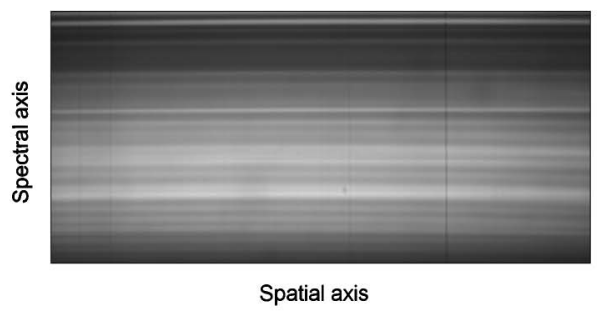

(e)

Fig. 4. (Color online) Hyperspectral image of the plasma column for a seam performed with (a) $26 \mathrm{~A}$ and (c) $50 \mathrm{~A}$. Spectral capture of a seam performed with (b) $26 \mathrm{~A}$ and (d) $50 \mathrm{~A}$. (e) Detail extracted from (c).

of $37 \mathrm{~A}$ and a protective gas flow rate of $12 \mathrm{l} / \mathrm{min}$. This rate was manually altered to simulate a perturbation on the gas flow, reducing it to approximately $12 \mathrm{l} / \mathrm{min}$ for $0.5 \mathrm{~s}$. A sequence of the images acquired by the hyperspectral imaging system is presented in Fig. $\underline{5}$; Figs. 5(a), 5(c), and 5(e) show the correct welding conditions, and Figs. $5(\mathrm{~b})$ and $5(\mathrm{~d})$ show the simulated defects. In this particular case the differences between the captured images are obvious, and, consequently, the defects could easily be identified. Spectra captured by means of the alternative spectroscopic setup are also shown in Figs. 5(f) (correct welding) and $5(\mathrm{~g})$ (gas flow perturbation). Figure 5(h) shows the plasma electronic temperature profile

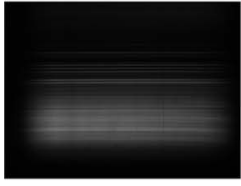

(a)

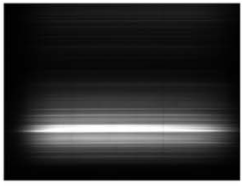

(b)

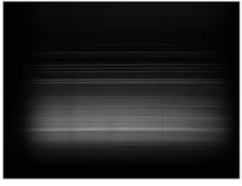

(c)

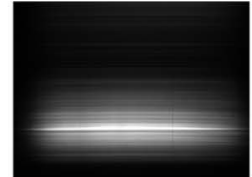

(d)

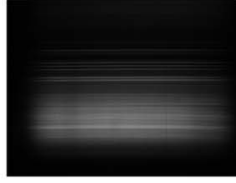

(e)

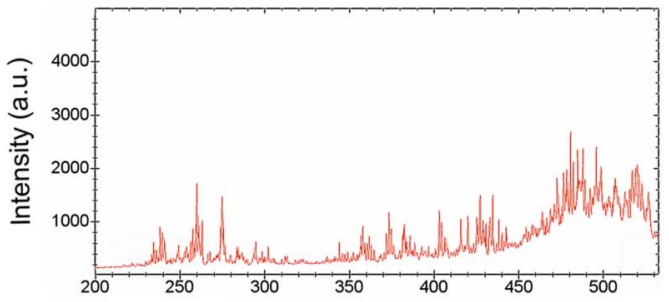

(f)

Wavelength $(\mathrm{nm})$
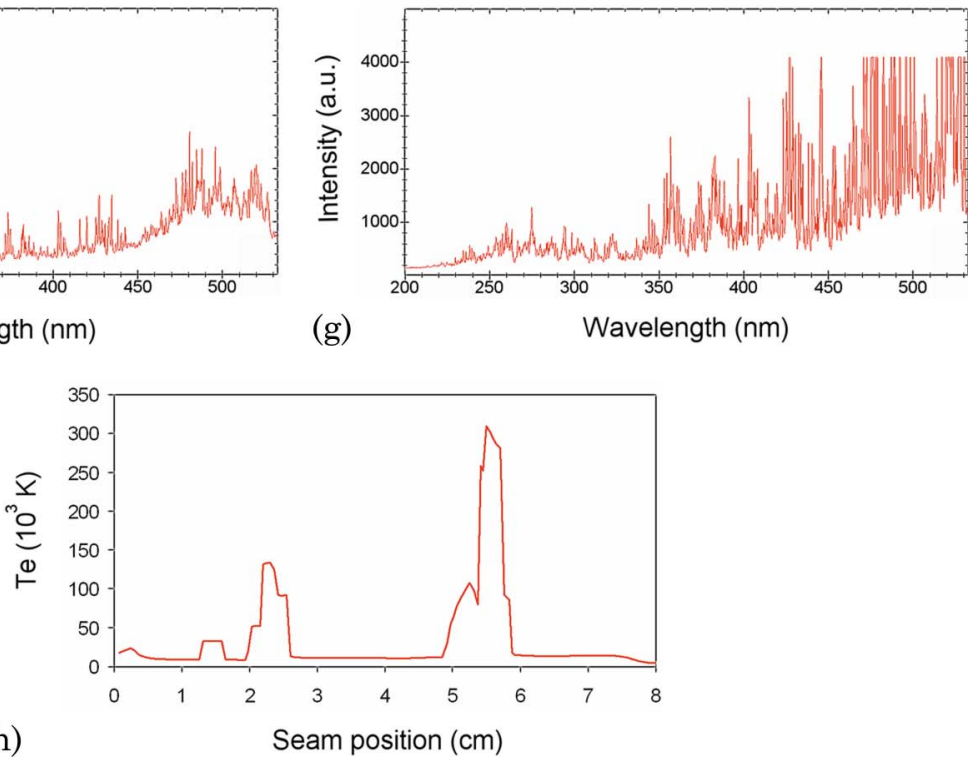

Fig. 5. (Color online) (a), (c), (e) Hyperspectral image of the plasma column for a seam performed with $37 \mathrm{~A}$ and a gas flow rate of 12 1/min; (b), (d) gas flow rate reduction; (f), (g) plasma spectra captured during standard and defective welding conditions; (h) plasma electronic temperature profile for the defective seam. 
determined with two Ar II emission lines (480.6 and $488 \mathrm{mn}$ ), where the two gas shortages can be clearly identified. It is worth noting that in this case the value of the electronic temperature is significant only in the sections that are free of defects, where it is of the order of $11000 \mathrm{~K}$. However, the temperatures for the defects are not reliable, given that the selected lines exhibit saturation.

A possible processing approach for the hyperspectral images (see Fig. 6) is used to carry out the analysis with the seam at the top [Fig. 7(a)] and with the seam at the bottom [Fig. 7(b)]. In this case the defect was caused by an incision performed in the plate to obtain a nonconstant thickness along the welding direction (the resulting plate thicknesses were 1.5 and $1 \mathrm{~mm}$ ). The spectra acquired by the imaging system were processed by means of the selection of the suitable region of interest (ROI), the extraction of the intensity plane of the resulting image. and the quantification of the average intensity for each captured hyperspectral image. Two results are depicted in Figs. 7(c) and 7(d), where the ROIs highlighted in Fig. $7 \overline{(\mathrm{e})}$ were employed. It can be appreciated that ROI 2 gives rise to a profile in which the correlation between the output signal and the defect is better. This result suggests, as already discussed, that a suitable selection of the ROI is needed to provide the required monitoring signal. It is also worth mentioning that the consideration of different materials and shielding gases might lead to a modification in the chosen ROIs. In this regard, a suitable estimation of the ROI geometry and location could allow use of a general ROI for different processes. This would imply the selection of a spectral range covering contributions from the different species under analysis.

\section{Spatial Analysis of the Plasma Column}

The addition of the spatial dimension in the hyperspectral images allowed us to carry out a detailed analysis of the plasma column in terms of the spectral response for each particular location. In this regard, a suitable selection of the most interesting

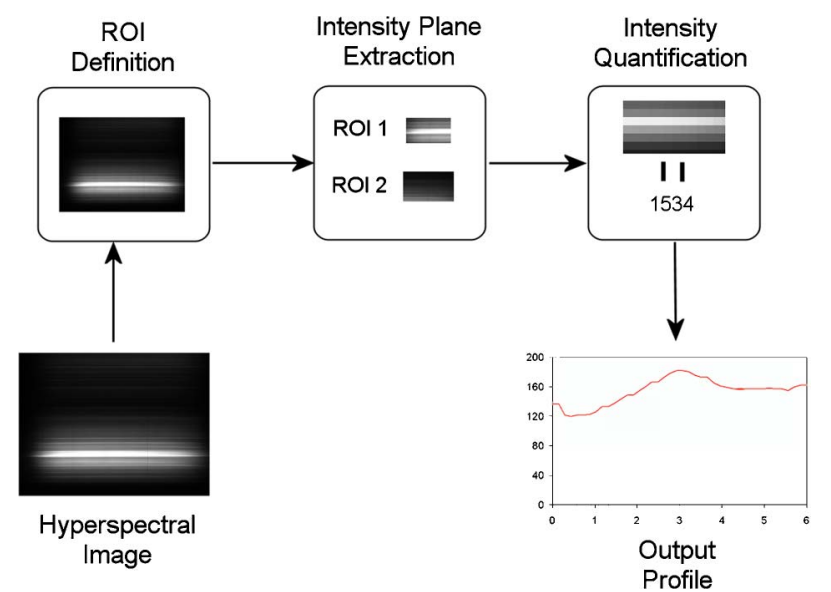

Fig. 6. (Color online) Image processing scheme proposed for generation of the output monitoring parameter. (a)

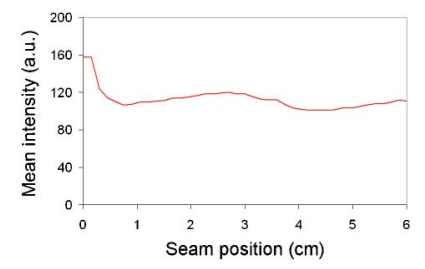

(c) (b)

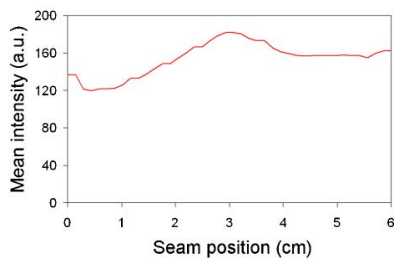

(d)

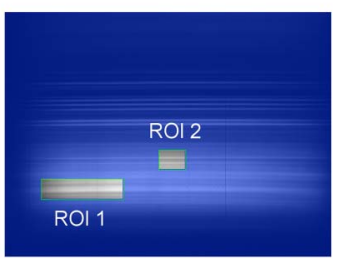

(e)

Fig. 7. (Color online) (a) Seam with incision at the top and (b) seam with incision at the bottom. (c), (d) Mean intensity profiles for ROI 1 and ROI 2, repectively; (e) selected ROIs for the acquired hyperspectral images.

spectral and spatial bands, in terms of their ability to discriminate among sound and defective welds, can be performed. Taking the experimental test presented in Fig. 7, it was previously discussed how the selection of the ROI affects the resulting monitoring parameter and, consequently, the performance of the designed system. A more specific analysis is shown in Fig. 8, where four different spatial locations within the plasma column have been chosen to identify an appropriate spectral band for the subsequent welding quality analysis. The outer regions of the plasma column are considered in Figs. 8(a) and 8(d), where the spectra for sound weld and gas shortages 1 and 2 are depicted. It can be appreciated how the spectral response is more intense for the second gas perturbation, since the response for the first is clearer in Fig. 8(d), where the spectra were formed as an average of pixels from 636 to 640 . The spectral band located at approximately $567 \mathrm{~nm}$ appears to be optimal in terms of the discrimination of this type of defect. Figures $8(\mathrm{~b})$ and 8 (c) show similar results, but the spectra associated with gas shortage 2 are saturated and, in addition, the slope of this signal for wavelengths higher than $800 \mathrm{~nm}$ could lead to false conclusions.

In Fig. 9 the spectra associated with the central region of the plasma column generated during the experimental test already discussed in Fig. 7 is analyzed. In this particular example, the differences among sound and defective spectra are rather subtle, which makes it difficult to find a suitable spectral region for the generation of the system output parameter. This is especially obvious in Figs. 7(c) and 7(d). It is worth noting that, without proper analysis of the spectra, the hyperspectral images give the impression that the first emission line, (lower 

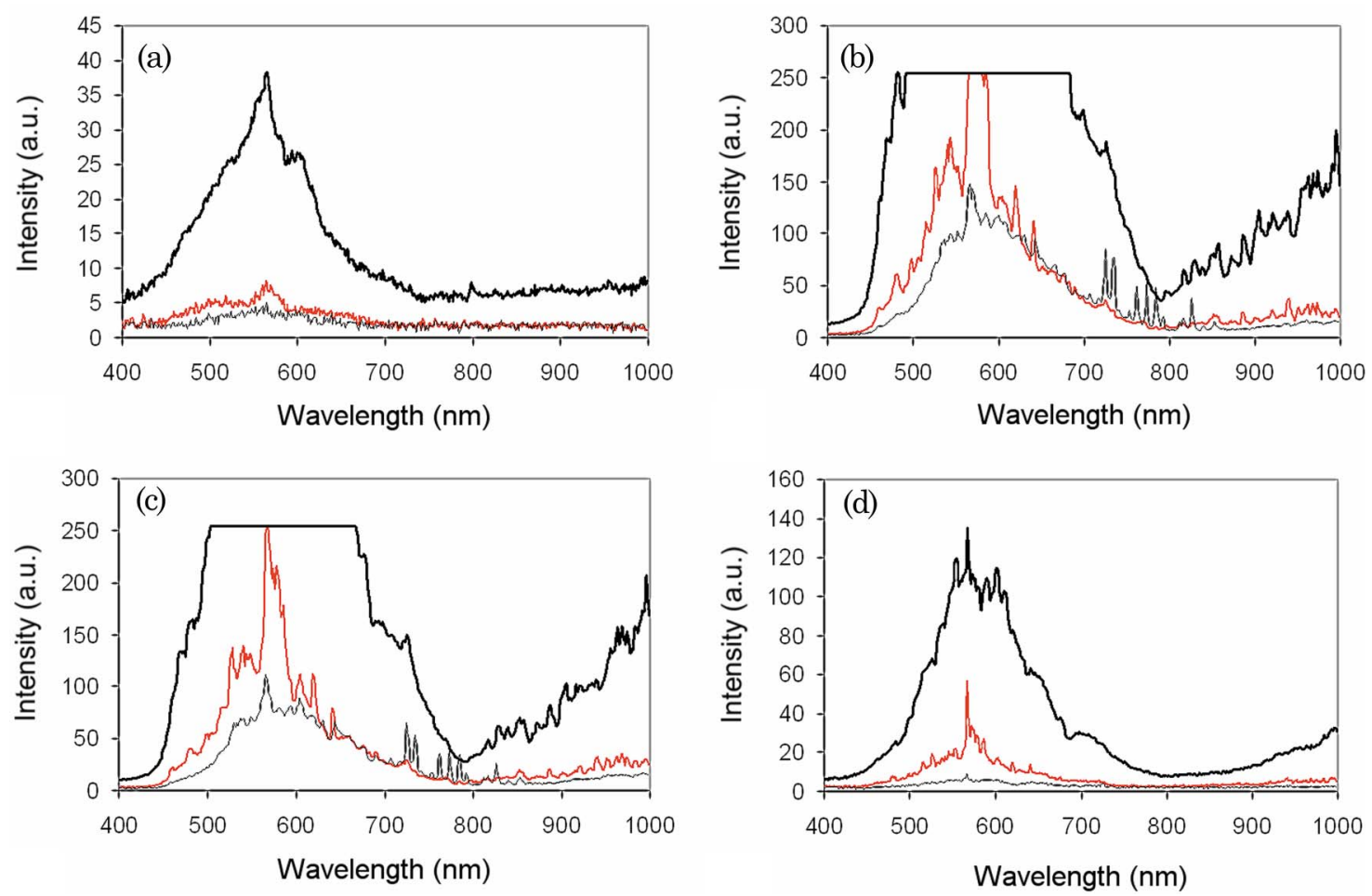

Fig. 8. (Color online) Seam with gas shortages and average spectral responses for (a) pixels 1 to 5 , (b) pixels 256 to 260 , (c) pixels 516 to 520, (d) pixels 636 to 640 .

wavelength) highlighted in Fig. $\underline{9}$ exhibits lower intensity for the defective area. However, this effect is caused by the higher contribution of the background radiation, as the total intensity for this

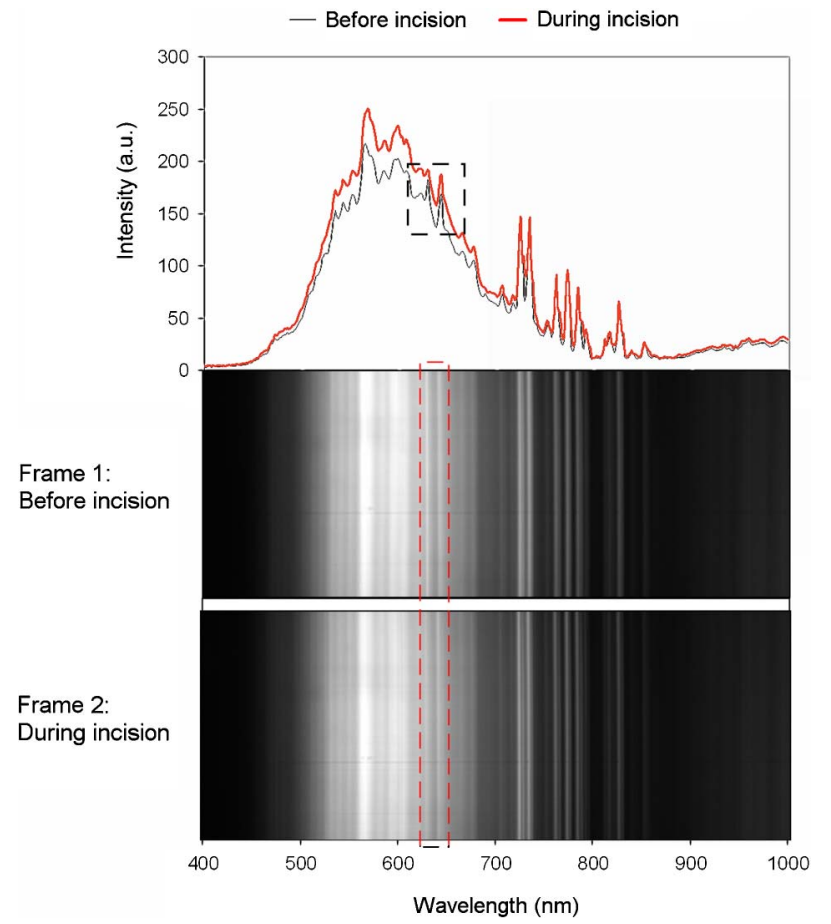

Fig. 9. (Color online) Spectra extracted from the hyperspectral images captured before (sound weld) and during the incision (defect) performed on a plate. specific wavelength (approximately $627 \mathrm{~nm}$ ) is in fact higher.

It is worth mentioning that this kind of study could also be used to perform an optimal spectral band selection for the traditional spectroscopic approach, where several emission lines are present in the spectra, but typically only two are chosen to determine $T_{e}$.

\section{Conclusions}

We have explored the use of imaging spectroscopy applied to online welding quality monitoring. In comparison with the traditional spectroscopic approach, the use of hyperspectral devices such as PGP-based spectrographs allowed us to capture detailed images of the plasma column with both spectral and spatial information. In this sense, the required analysis to produce an efficient output monitoring parameter does not require the identification of the plasma emission lines, which is costly in terms of the computational performance of the whole system. By means of several experimental tests it has been demonstrated that simple image processing strategies can provide the desired results, allowing us to identify defective regions in a given seam.

Although a more extensive study with industrial samples should be conducted, including different materials and shielding gases as well as different types of weld flaw, we believe that, in spite of the higher cost of the imaging spectroscopy system in comparison with the traditional CCD spectrometer approach, this solution could be successfully implemented in 
industrial welding applications. In addition, the vast amount of information acquired by the system permitted us to carry out a detailed analysis of the plasma spectral response for different spatial locations, which gives rise to useful information in terms of spectral band selection to develop optimal discrimination between sound and defective welds.

Analysis of industrial samples with a large set of defects is being conducted in an attempt to establish the validity of the proposed solution for real industrial applications. On the other hand, the proposed image processing scheme could be improved, and it would be interesting to try to define a general ROI that would be equally valid for different welding processes. It should also be determined whether a sensor fusion approach would be worth exploring within this framework.

This research has been co-supported by the Science and Technology Ministry of the Spanish Government through the CICYT TEC2005-08218-C0202 and TEC2007-67987-C02-01 projects.

\section{References}

1. G. Müller, E. Rohde, J. Beuthan, K. Dörschel, U. Bindig, and H. G. Eberle, "Optoelectronic diagnostics in biomedical engineering," Laser Phys. 13, 196-206 (2003).

2. W. B. Grant, R. H. Kagann, and W. A. McClenny, "Optical remote measurement of toxic gases," J. Air Waste Manage. Assoc. 42, 18-30 (1992).

3. V. Wagner, D. Drews, N. Esser, D. R. T. Zahn, J. Geurts, and W. Richter, "Raman monitoring of semiconductor growth," J. Appl. Phys. 75, 7330-7333 (1994).

4. P. Vandenabeele, H. G. M. Vandenabeele, and L. Moens, "A decade of Raman spectroscopy in art and archaeology," Chem. Rev. 107, 675-686 (2007).

5. B. A. Weinstock, J. Janni, L. Hagen, and S. Wright "Prediction of oil and oleic acid concentrations in individual corn (Zea mays L.) kernels using near-infrared reflectance hyperspectral imaging and multivariate analysis," Appl. Spectrosc. 60, 9-16 (2006).

6. K. C. Lawrence, W. R. Windham, B. Park, D. P. Smith, and G. H. Poole, "Comparison between visible/NIR spectroscopy and hyperspectral imaging for detecting surface contaminants on poultry carcasses," Proc. SPIE 5271, 35-42 (2004).

7. A. Plaza, D. Valencia, and J. Plaza, "An experimental comparison of parallel algorithms for hyperspectral analysis using heterogeneous and homogeneous networks of workstations," Parallel Comput. 34, 92-114 (2008).

8. B. Park, K. C. Lawrence, W. R. Windham, D. P. Smith, and P. W. Feldner, "Hyperspectral imaging for food processing automation," Proc. SPIE 4816, 308-316 (2002).
9. P. B. Garcia-Allende, O. M. Conde, J. Mirapeix, A. Cobo, and J. M. Lopez-Higuera, "Quality control of industrial processes by combining a hyperspectral sensor and Fisher's linear discriminant analysis," Sens. Actuators B 129, 977-984 (2008).

10. L. Grad, J. Grum, I. Polajnar, and J. M. Slabe, "Feasibility study of acoustic signals for on-line monitoring in short circuit gas metal arc welding," Int. J. Mach. Tools Manuf. 44, 555-561 (2004)

11. G. J. Zhang, Z. H. Yan, and L. Wu, "Visual sensing of weld pool in variable polarity TIG welding of aluminium alloy," Trans. Nonferr. Met. Soc. China 16, 522-526 (1997).

12. A. Al-Habaibeh and R. Parkin, "An autonomous low-cost infrared system for the on-line monitoring of manufacturing processes using novelty detection," Int. J. Adv. Manuf. Technol. 22, 249-258 (2003).

13. A. Ancona, V. Spagnolo, P. M. Lugara, and M. Ferrara, "Optical sensor for real-time monitoring of $\mathrm{CO}_{2}$ laser welding process,” Appl. Opt. 40, 6019-6025 (2001).

14. P. Sforza and D. de Blasiis, "On-line optical monitoring system for arc welding," NDT \& E Int. 35, 37-43 (2002).

15. H. R. Griem, "Validity of local thermal equilibrium in plasma spectroscopy," Phys. Rev. 131, 1170-1176 (1963).

16. G. N. Haddad and A. J. D. Farmer, "Temperature determinations in a free-burning arc: 1. Experimental techniques and results in argon," J. Phys. D 17, 1189-1196 (1984).

17. G. N. Haddad, A. J. D. Farmer, P. Kovitya, and L. E. Cram, "Physical processes in gas-tungsten arcs," IEEE Trans. Plasma Sci. 14, 333-336 (1986).

18. J. F. Bott, "Spectroscopic measurement of temperatures in an argon plasma arc," Phys. Fluids 9, 1540-1547 (1966).

19. A. Marotta, "Determination of axial thermal plasma temperatures without Abel inversion," J. Phys. D 27, 268-272 (1993).

20. J. Mirapeix, A. Cobo, C. Jaúregui, and J. M. López-Higuera, "Fast algorithm for spectral processing with application to on-line welding quality assurance," Meas. Sci. Technol. 17, 2623-2629 (2006).

21. J. Mirapeix, A. Cobo, S. Fernandez, R. Cardoso, and J. M. Lopez-Higuera, "Spectroscopic analysis of the plasma continuum radiation for on-line arc-welding defect detection," J. Phys. D 41, 135202 (2008).

22. J. Mirapeix, A. Cobo, A. Quintela, and J. M. Lopez-Higuera, "Embedded spectroscopic fiber sensor for on-line arc-welding analysis," Appl. Opt. 46, 3215-3220 (2007).

23. J. A. Aguilera and C. Aragon, "Characterization of a laserinduced plasma by spatially resolved spectroscopy of neutral atom and ion emissions. Comparison of local and spatially integrated measurements," Spectrochim. Acta Part B 59 1861-1876 (2004).

24. T. Vaarala, M. Aikio, and H. Keraenen, "An advanced prismgrating-prism imaging spectrograph in on-line industrial applications," Proc. SPIE 3101, 322-330 (1997). 